\title{
Clima familiar deportivo y autoconcepto físico en la adolescencia
}

\author{
Lorena Revuelta e Igor Esnaola \\ Universidad del País Vasco (España)
}

\begin{abstract}
En este estudio se analizan las relaciones entre las actitudes, valores e intereses familiares percibidos sobre la actividad físico-deportiva en general y de los hijos e hijas en particular, la autopercepción física y la actividad físico-deportiva realizada por los adolescentes. Participaron en el estudio 355 adolescentes de entre 12 y 22 años de las comunidades autónomas vasca y cántabra, a quienes se administraron el Cuestionario de Clima Familiar Deportivo y el Cuestionario de Autoconcepto Físico. Los resultados indican que un clima familiar deportivo favorable se relaciona con un autoconcepto físico más elevado y con una mayor frecuencia de actividad físico-deportiva. Así mismo, aparecen diferencias en cuanto al clima familiar deportivo en función del sexo de los participantes. Las chicas percibieron climas menos favorables que los chicos y se advirtieron diferencias a este respecto en función del sexo del resto de hijos e hijas de la unidad familiar. Los hijos de familias en las que sólo había chicos percibieron climas familiares deportivos más positivos que aquellos pertenecientes a familias en las que había chicos y chicas, siendo las percepciones más desfavorables las de las chicas que sólo tenían hermanas o eran hijas únicas.
\end{abstract}

Palabras clave: Clima familiar deportivo, autoconcepto físico, actividad físico-deportiva, otros significativos, adolescencia.

Family sports environment and self-concept during adolescence. The purpose of the present study was to analyze the relationship between the whole family attitudes, values and interests toward sport and physical activity, and the ones of the children of the family (boys and girls). 355 adolescents age 12 to 22 from the Spanish regions of Basque Country and Cantabria took part in the study. There were administered the Family Sports Environment Questionnaire along with the Physical Self-Concept Questionnaire Scores derived from the questionnaires show a relationship between a good family sports environment and a higher physical self-concept and a more frequent practice of sports in adolescents. Differences on the family sports environment have been found depending on the gender of the studied adolescent. Girls perceived a worse family sports environment than boys. Also different perceptions were noticed depending on the gender of the other children of the family: the boys of families who only have sons perceived more positive environment than the boys of families with children of both genders. The worst perceptions were the ones of the girls who were the only child or who only had female siblings.

Key words: Family sports environment, physical self-concept, sport and physical activity, significant others, adolescence. 
La adolescencia es una de las etapas más importantes y decisivas en lo que respecta al estudio e intervención sobre el autoconcepto. Durante la adolescencia media se produce la recomposición del autoconcepto del adolescente una vez superada la crisis que experimenta durante la adolescencia temprana (Brettschneider y Heim, 1997). En esta etapa, como resultado de las influencias del medio social y cultural en el que se desenvuelven y de la evolución de sus experiencias y representaciones personales (Horn y Harris, 1996), los adolescentes elaboran formas más complejas e integradas de autorepresentación que una vez consolidadas presentan una considerable estabilidad (Esteve, 2005). Es además en esta etapa, cuando comienzan a surgir las primeras diferencias en el autoconcepto debidas al sexo (Amezcua y Pichardo, 2001; Esnaola, 2009; Horn y Harris, 1996).

Numerosos estudios confirman una relación entre el nivel de actividad físicodeportiva y el nivel de autoconcepto físico en edades diferentes (Esnaola y Zulaika, 2009; Goñi y Zulaika, 2000; Infante y Goñi, 2009). Parece que dentro del sistema jerárquico de dominios y subdominios del autoconcepto, la autopercepción física tiene una especial significatividad y relevancia. En este sentido, la mejora de los subdominios específicos constituyentes del autoconcepto físico, habilidad física, condición física, fuerza y atractivo a través de la actividad físico-deportiva, tendría un efecto positivo en éste y en consecuencia en el autoconcepto general (Goñi, Ruiz de Azúa y Rodríguez, 2004). Además, el propio autoconcepto físico se considera una variable relacionada con la actividad físico-deportiva, con lo que mejoras en éste redundarían en mayores probabilidades de iniciar o continuar practicando un deporte o actividad física (Moreno, Moreno y Cervelló, 2007). A partir de estas premisas, numerosas investigaciones han sido llevadas a cabo al respecto terminando por aceptarse una relación circular y recíproca entre autoconcepto físico y actividad físico-deportiva (Boyd y Hrycaiko, 1997; Fox, 2000).

A la hora de estudiar y diseñar estrategias de intervención destinadas al incremento de los niveles de actividad físico-deportiva en las etapas tempranas de la vida, es fundamental abordar aquellos aspectos del adolescente y el medio en que se desenvuelve, decisivos para la elección de este tipo de actividades, como su círculo social y muy especialmente la familia (Esteve, Musitu y Lila, 2005; Horn y Horn, 2007). Estos otros significativos configuran en torno al chico un clima de apoyo o de desinterés por la actividad físico-deportiva.

El clima familiar deportivo comprende una serie de comportamientos y actitudes más o menos positivas que padres y madres mantienen hacia el deporte y la actividad física (Esteve, 2005) en general, y la actividad físico-deportiva de sus hijos en particular; condicionando la forma de entender y vivir el deporte de éstos. Así, el clima familiar deportivo se relacionaría con ciertas características de la experiencia deportiva de los adolescentes, como el valor e importancia asignado a este tipo de actividades, el nivel de compromiso con las mismas o las autopercepciones específicas (Brustad, 1992; Esteve et al., 2005).

Uno de los papeles principales que el clima familiar deportivo atribuye a los padres y madres es el de proveedores de experiencias deportivas. Se valora su propia actividad físico-deportiva, la importancia dada a su actividad y la de sus hijos, y los 
materiales y situaciones físico-deportivas que proporcionan a éstos. Al posibilitar o no el contacto directo o indirecto de los adolescentes con el deporte y la actividad física, no sólo facilitan o no el acceso a los mismos e incrementan o no su nivel de participación, sino que transmiten mensajes de valoración directa e indirecta de estas actividades. Esta valoración tiene repercusión en el valor que chicos y chicas le atribuyen a la actividad físico-deportiva, con las consecuencias directas que esta valoración tiene sobre su propia actividad (Eccles y Harold, 1991; Fredricks y Eccles, 2004).

Más aún, proporcionando momentos de ejercitación, padres y madres permiten también que los adolescentes evalúen, pongan en práctica y mejoren sus capacidades, mejorando sus propias percepciones físicas (Harter, 1986), y posiblemente su autoconcepto general y la frecuencia de actividad físico-deportiva, alimentando un círculo con beneficios potenciales y reales sobre la salud física y psicológica de sus hijos (Pérez y Devís, 2003). Además, siendo el deporte juvenil una actividad que a menudo se presta a una intensa participación por parte de los padres en una variedad de papeles, es posible un contacto inmediato de padres e hijos y una oportunidad de proporcionar un feedback inmediato y específico que contribuiría a la formación de las autopercepciones físicas (Fredericks y Eccles, 2004). No sólo eso, muchos autores relacionan al propio clima familiar deportivo con el autoconcepto físico de los adolescentes de forma directa (Brustad, 1996; Esteve, 2005; Rodríguez y Beato, 2002; Weiss, 2000).

Al hablar de socialización familiar, un aspecto vital a considerar es el sexo de los hijos. Tal y como se ha confirmado en diferentes investigaciones, padres y madres proporcionan actividades, materiales y estímulos diferentes a sus hijos creando climas motivacionales diferentes en función del sexo (Brustad, 1996; Roberts, 1995; Eccles y Harold, 1991). Aparecen también diferencias en la valoración que estos hacen de la actividad en cuestión según sean sus hijos o hijas quienes participen (Brustad, 1993; Eccles, Jacobs y Harold, 1990; Fredericks y Eccles, 2005; Horn y Horn, 2007; Jacobs y Eccles, 1992).

Es importante destacar por tanto que, teniendo en cuenta que los otros significativos permiten en ocasiones que aspectos como sus creencias o el sexo de sus hijos influyan en sus interpretaciones y en las actividades a las que les invitan, pueden contribuir a que emerjan diferencias en las autopercepciones, expectativas y percepciones sobre las actividades (Eccles y Harold, 1991; Fredericks y Eccles, 2005) que podrían explicar las diferencias significativas que a menudo se registran en la participación o el abandono deportivo, la valoración de la actividad físico-deportiva o el autoconcepto físico a favor de los chicos (Esnaola, 2009).

Teniendo en cuenta lo expuesto anteriormente, el propósito de este estudio es analizar las relaciones entre el clima familiar deportivo percibido por los adolescentes, su autoconcepto físico y su nivel de actividad físico-deportiva, evaluando si estas relaciones varían en función del sexo de los participantes.

Además, teniendo en cuenta que, pese a que padres y madres se relacionan de forma directa con cada uno de sus hijos, en general suelen conformar un modelo consistente de comportamientos y actitudes hacia ellos de manera global (Forehan y Nousiainen, 1993; Sallay, 2000), analizaremos la percepción de la actitud paterno/materna hacia la actividad físico-deportiva valorando la influencia que en esta 
actitud pudiera tener el sexo de la totalidad de hijos de la unidad familiar. Este tipo de percepciones ha sido analizada hasta ahora en función del sexo del hijo/a encuestado (Esteve, 2005; Esteve et al., 2005); nuestro trabajo las analizará en función del sexo de los hijos de la familia con el objetivo de determinar si es verdaderamente el sexo de los hijos e hijas lo que influye en las actitudes/comportamiento de los padres y no el sexo del hijo/a entrevistado lo que determina estas diferencias.

\section{METODO}

\section{Participantes}

Participaron en el estudio 355 adolescentes pertenecientes a dos institutos públicos y un instituto privado y dos facultades de la comunidad autónoma cántabra y del País Vasco seleccionados por conveniencia y contactados a través de sus respectivos directores y jefes de estudios, que mostraron su conformidad a participar en el estudio una vez conocidos los objetivos y procedimientos del mismo. La media de edad de los participantes fue de 17.87 años $(D T=2.97)$. En cuanto a la distribución por sexos, 142 (40\%) fueron chicos y $213(60 \%)$ chicas.

\section{Instrumentos}

Los participantes completaron los siguientes instrumentos, cuyo contenido forma parte de un estudio más amplio:

Cuestionario de Autoconcepto Físico (CAF) (Goñi, Ruiz de Azúa y Rodríguez, 2006). Este cuestionario consta de 36 ítems y seis escalas: habilidad física, condición física, atractivo físico, fuerza, autoconcepto físico general y autoconcepto general. Seis ítems redactados de forma enunciativa fueron empleados para cada escala planteándose para las respuestas cinco opciones en una escala tipo Likert. En cuanto a las propiedades psicométricas del cuestionario, hay que señalar que se utilizó el Alpha de Cronbach para la medida de la consistencia interna, con unos resultados de $\alpha=.86$ para el autoconcepto físico general; $\alpha=.84$ para la habilidad física; $\alpha=.88$ para la condición física; $\alpha=.87$ para el atractivo físico; $\alpha=.83$ para la fuerza física; $\mathrm{y}, \alpha=.84$ para el autoconcepto general.

Escala de Clima Familiar Deportivo (CFD) (Escartí, García-Ferriol y Musitu, 1993). Esta escala está compuesta de 14 ítems destinados a valorar el apoyo paterno y materno hacia la práctica de actividad física y/o deportiva de los hijos, los ejemplos cotidianos, el apoyo explícito, la proporción de material deportivo y la preocupación por el ambiente en que esta práctica se desarrolla. Pese a que los creadores del constructo clima familiar deportivo subdividieron al mismo en cuatro factores para su estudio y aplicación (apoyo, comunicación, educación e interés), en ciertos trabajos se ha utilizado el mismo como una dimensión global en la que se valoran actitudes paterno/maternas globales más o menos positivas hacia el deporte en general y la actividad físicodeportiva de sus hijos/as en particular (Esteve et al., 2005). En el estudio que presentamos también se ha utilizado esta escala global. Las respuestas son registradas 
también mediante una escala tipo Likert cuya modalidad de respuesta es una dimensión continua desde el 0 (completamente en desacuerdo) hasta el 100 (completamente de acuerdo). Los datos psicométricos de la escala indican una consistencia interna de $\alpha=$ .77 .

Cuestionario sobre Actividad Físico-Deportiva. Elaborado específicamente para el estudio, registra la actividad físico-deportiva de los sujetos y la frecuencia. Se preguntó a los participantes si realizaban actividad física y/o deporte y por su frecuencia de práctica, proporcionando las siguientes opciones de respuesta: a) Nunca; b) de vez en cuando; c) una vez a la semana como mínimo; d) entre dos y tres días por semana; y, d) más de cuatro días por semana. Tomando como base la clasificación de Román et al., en el Estudio Enkid (2006) según la cual aquellas personas que practican actividades de corte físico-deportivo con una frecuencia menor a tres días semanales son consideradas inactivas o sedentarias, se decidió dividir a los sujetos en esas dos categorías, activo y sedentario. En el primer grupo se incluyeron a aquellos que dijeron participar entre dos y tres días o más de cuatro días por semana; y en el segundo, a los que respondieron con una de las tres primeras opciones.

\section{Procedimiento}

Una vez obtenido el permiso por parte del centro para la realización del estudio, el cuestionario, cuya realización llevó entre 30 y 50 minutos en función de la edad de los participantes, fue administrado al alumnado durante las horas de tutoría o educación física según la disponibilidad de los distintos docentes, por un investigador que permanecía en el aula durante todo el proceso para solucionar las posibles dudas o problemas que surgieran y recogía los cuestionarios en el momento de su finalización. Se insistió en la inexistencia de respuestas correctas o incorrectas, y se aseguraron la confidencialidad y anonimato en el tratamiento de los datos.

\section{Análisis de datos}

El análisis de los datos fue realizado utilizando el paquete estadístico SPSS 17.0 para Windows, ejecutándose todos los procedimientos estadísticos a un nivel de confianza del 95\% (nivel de significación de .05). Se realizaron las oportunas pruebas para analizar la homocedasticidad, normalidad e independencia observando que las escalas cumplían las condiciones paramétricas.

Con objeto de analizar la relación entre el autoconcepto físico y el clima familiar deportivo y la frecuencia de actividad físico-deportiva se empleó la prueba $\mathrm{T}$ de student. Se llevó a cabo también una correlación entre el clima familiar deportivo y el autoconcepto físico mediante el método Pearson. Teniendo en cuenta el hecho de que estudios previos han encontrado diferencias en las variables analizadas entre sexos (Esnaola, 2009; Esteve, 2005; Esteve et al., 2005; Rodríguez y Beato, 2002), se realizaron los análisis de forma independiente en cada una de las muestras (chicos y chicas). 
Finalmente, para analizar las puntuaciones en la escala de clima familiar deportivo teniendo en cuenta el sexo de todos los hijos de la familia, se procedió a realizar un análisis de la varianza factorial (ANOVA) empleando los grupos Sólo hijos, Sólo hijas e Hijos e hijas. Una vez obtenidos los resultados del ANOVA, se ejecutaron las posteriores comparaciones múltiples (post-hoc) de Tukey.

\section{RESULTADOS}

La siguiente tabla recoge la distribución de la muestra así como el análisis de la frecuencia de actividad:

Tabla 1. Distribución de la muestra en función de la frecuencia de la actividad físico-deportiva

\begin{tabular}{lcccc}
\hline & \multicolumn{3}{c}{ Frecuencia/Actividad } \\
\cline { 2 - 6 } Sexo & Hombre & Mujer & Hombre & Mujer \\
\hline Muestra & 112 & 108 & 30 & 103 \\
\hline Porcentaje & $78.9 \%$ & $51.2 \%$ & $21.1 \%$ & $48.8 \%$ \\
\hline Total & \multicolumn{3}{c}{221} & \multicolumn{3}{c}{134} \\
\hline Porcentaje del total & \multicolumn{2}{c}{$62.3 \%$} & \multicolumn{3}{c}{$37.7 \%$} \\
\hline
\end{tabular}

Al analizar la frecuencia, 221 adolescentes fueron calificados como activos frente a 134 que por sus respuestas se incluyeron en el grupo de sedentarios. Se encontraron diferencias estadísticamente significativas $(p<.000)$ a favor de los varones. En el grupo activo 112 (78.9\%) fueron chicos frente a las 108 (51.2\%) chicas; mientras que en el grupo de sedentarios sólo $30(21.1 \%)$ fueron chicos frente a las 103 chicas $(48.8 \%)$.

El análisis del autoconcepto físico en función del sexo de los sujetos que podemos observar en la tabla 2 confirmó los siguientes datos:

Tabla 2. Puntuaciones en las escalas del CAF en función del sexo

\begin{tabular}{lcccc}
\hline & Sexo & $N$ & $M d$ & $S D$ \\
\hline \multirow{2}{*}{ Autoconcepto físico general } & Hombre & 142 & 24.92 & 4.21 \\
\cline { 2 - 5 } & Mujer & 211 & 21.13 & 5.34 \\
\hline \multirow{2}{*}{ Habilidad física } & Hombre & 142 & 23.67 & 3.83 \\
\cline { 2 - 5 } & Mujer & 211 & 19.88 & 4.77 \\
\hline \multirow{2}{*}{ Condición física } & Hombre & 142 & 23.30 & 4.55 \\
\cline { 2 - 5 } & Mujer & 211 & 17.94 & 4.96 \\
\hline \multirow{2}{*}{ Atractivo físico } & Hombre & 142 & 22.22 & 4.28 \\
\cline { 2 - 5 } & Mujer & 211 & 20.21 & 5.05 \\
\hline \multirow{2}{*}{ Fuerza } & Hombre & 142 & 20.05 & 4.58 \\
\cline { 2 - 5 } & Mujer & 211 & 16.59 & 5.05 \\
\hline \multirow{2}{*}{ Autoconcepto general } & Hombre & 142 & 25.56 & 3.46 \\
\cline { 2 - 5 } & Mujer & 211 & 24.20 & 3.97 \\
\hline
\end{tabular}


Aparecieron diferencias estadísticamente significativas en función del sexo en todas las escalas a favor de los varones: habilidad $\left(t_{(353)}=7.886, p=.000\right)$, condición $\left(t_{(353)}=10.398, p=.000\right)$, atractivo $\left(t_{(353)}=4.017, p=.000\right)$, fuerza $\left(t_{(353)}=6.643, p=\right.$ $.000)$, autoconcepto físico general $\left(t_{(353)}=7.103, p=.000\right)$, y autoconcepto general $\left(t_{(353)}\right.$ $=3.296, p=.001)$. Los adolescentes varones se percibieron mejor que las chicas en todos los subdominios del autoconcepto físico, así como en las dos escalas superiores.

En lo que respecta a los análisis que relacionan el autoconcepto físico y la frecuencia de actividad físico-deportiva (Tabla 3), en la muestra masculina se encontraron diferencias estadísticamente significativas en todas las escalas a favor del grupo activo: habilidad $\left(t_{(142)}=3.587, p=.000\right)$, condición $\left(t_{(142)}=7.430, p=.000\right)$, atractivo $\left(t_{(142)}=3.375, p=.001\right)$, fuerza $\left(t_{(142)}=3.578, p=.000\right)$, autoconcepto físico general $\left(t_{(142)}=4.280, p=.000\right)$, y autoconcepto general $\left(t_{(142)}=4.621, p=.000\right)$. Es decir, los chicos activos, se percibieron mejor que los sedentarios en todos los subdominios del autoconcepto físico, así como en las dos escalas superiores.

Asimismo, en la muestra femenina, aparecieron diferencias estadísticamente significativas en las siguientes escalas a favor del grupo activo: habilidad física $\left(t_{(211)}=\right.$ $5.238, p=.000)$, condición física $\left(t_{(211)}=5.692, p=.000\right)$, fuerza $\left(t_{(211)}=2.934, p=\right.$ $.004)$, autoconcepto físico general $\left(t_{(211)}=2.549, p=.012\right)$, y autoconcepto general $(t$ $(211)=2.800, p=.006)$.

Tabla 3. Puntuaciones en las escalas del CAF en función de la frecuencia

\begin{tabular}{|c|c|c|c|c|c|}
\hline & Sexo & Práctica & $N$ & $M D$ & $D T$ \\
\hline \multirow{4}{*}{ Autoconcepto físico general } & \multirow{2}{*}{ Hombre } & Activo & 112 & 25.66 & 3.32 \\
\hline & & Sedentario & 30 & 22.16 & 5.85 \\
\hline & \multirow{2}{*}{ Mujer } & Activo & 109 & 22.04 & 5.09 \\
\hline & & Sedentario & 104 & 20.19 & 5.45 \\
\hline \multirow{4}{*}{ Habilidad física } & \multirow{2}{*}{ Hombre } & Activo & 112 & 24.25 & 3.22 \\
\hline & & Sedentario & 30 & 21.53 & 5.05 \\
\hline & \multirow{2}{*}{ Mujer } & Activo & 109 & 21.45 & 4.34 \\
\hline & & Sedentario & 104 & 18.21 & 4.65 \\
\hline \multirow{4}{*}{ Condición física } & \multirow{2}{*}{ Hombre } & Activo & 112 & 24.52 & 3.27 \\
\hline & & Sedentario & 30 & 18.46 & 5.63 \\
\hline & \multirow{2}{*}{ Mujer } & Activo & 109 & 19.70 & 4.69 \\
\hline & & Sedentario & 104 & 16.08 & 4.56 \\
\hline \multirow{4}{*}{ Atractivo físico } & \multirow{2}{*}{ Hombre } & Activo & 112 & 22.83 & 3.75 \\
\hline & & Sedentario & 30 & 19.96 & 5.34 \\
\hline & \multirow{2}{*}{ Mujer } & Activo & 109 & 20.52 & 4.76 \\
\hline & & Sedentario & 104 & 19.88 & 5.34 \\
\hline \multirow{4}{*}{ Fuerza } & \multirow{2}{*}{ Hombre } & Activo & 112 & 20.72 & 4.04 \\
\hline & & Sedentario & 30 & 17.44 & 5.58 \\
\hline & \multirow{2}{*}{ Mujer } & Activo & 109 & 17.57 & $\overline{5.34}$ \\
\hline & & Sedentario & 104 & 15.57 & 4.54 \\
\hline \multirow{4}{*}{ Autoconcepto general } & \multirow{2}{*}{ Hombre } & Activo & 112 & 26.21 & 2.72 \\
\hline & & Sedentario & 30 & 23.13 & 4.73 \\
\hline & \multirow{2}{*}{ Mujer } & Activo & 108 & 24.94 & 3.64 \\
\hline & & Sedentario & 103 & 23.43 & 4.17 \\
\hline
\end{tabular}


La tabla 4 que observamos a continuación, presenta las correlaciones de Pearson entre el clima familiar deportivo y el autoconcepto físico.

Tabla 4. Correlaciones entre el clima familiar deportivo y las escalas del CAF

\begin{tabular}{cccccccc}
\hline & $\begin{array}{c}\text { Autoconcepto } \\
\text { físico }\end{array}$ & $\begin{array}{c}\text { Habilidad } \\
\text { física }\end{array}$ & $\begin{array}{c}\text { Condición } \\
\text { física }\end{array}$ & $\begin{array}{c}\text { Atractivo } \\
\text { físico }\end{array}$ & Fuerza & $\begin{array}{c}\text { Autoconcepto } \\
\text { general }\end{array}$ \\
\hline $\begin{array}{c}\text { CFD } \\
\text { Hombres }\end{array}$ & $\begin{array}{c}\mathrm{r} \text { de } \\
\text { Pearson }\end{array}$ & $.288^{* *}$ & $.342^{* *}$ & $.367 * *$ & $.328^{* *}$ & $.280^{* *}$ & $.210^{*}$ \\
\hline $\begin{array}{c}\text { CFD } \\
\text { Mujeres }\end{array}$ & $\begin{array}{c}\mathrm{r} \text { de } \\
\text { Pearson }\end{array}$ & $.193^{* *}$ & $.437 * *$ & $.430^{* *}$ & .030 & $.359^{* *}$ & $.245^{* *}$ \\
\hline
\end{tabular}

CFD: Clima Familiar Deportivo

Fueron registradas relaciones positivas y significativas $(p<.05)$ entre el clima familiar deportivo y todos los subdominios del autoconcepto físico, así como con las dos escalas generales, tanto en la muestra masculina como en la femenina (salvo en el atractivo físico).

Las diferencias en la percepción del clima familiar deportivo fueron más positivas entre el grupo de chicos que en el de chicas $\left(t_{(353)}=5.940, p=.000\right)$. Los análisis presentados por la tabla 5 muestran las diferencias en la percepción del clima familiar deportivo entre las chicas activas us. sedentarias fueron también estadísticamente significativas $\left(t_{(211)}=4.718, p=.000\right)$.

Tabla 5. Clima familiar deportivo en función de la frecuencia de actividad física

\begin{tabular}{rrlrrr}
\hline & Sexo & Práctica & \multicolumn{1}{c}{$N$} & \multicolumn{1}{c}{$M D$} & \multicolumn{1}{c}{$D T$} \\
\hline \multirow{3}{*}{ Clima familiar deportivo } & \multirow{2}{*}{ Hombre } & Activo & 112 & 886.12 & 200.06 \\
\cline { 2 - 6 } & & Sedentario & 30 & 808.92 & 187.78 \\
\cline { 2 - 6 } & \multirow{2}{*}{ Mujer } & Activo & 108 & 802.40 & 210.38 \\
\cline { 2 - 6 } & & Sedentario & 103 & 662.91 & 218.72 \\
\hline
\end{tabular}

Con objeto de determinar la importancia del sexo de la totalidad de hermanos en la actitud paterna hacia la actividad físico-deportiva se realizó un análisis de la varianza de un factor (ANOVA) para evaluar si existían diferencias en cuanto al clima familiar deportivo entre las familias en las que sólo había chicos, sólo había chicas o había chicos y chicas (Tabla 6).

Tabla 6. Clima familiar deportivo en función del sexo de los hermanos/as

\begin{tabular}{lllccc}
\hline & Sexo de los hermanos & $N$ & $M D$ & $D T$ & Tukey (a) \\
\hline \multirow{2}{*}{$\begin{array}{l}\text { Clima familiar } \\
\text { deportivo }\end{array}$} & Sólo chicos & 80 & 872.05 & 205.58 & \\
\cline { 2 - 5 } & Sólo chicas & 104 & 724.27 & 223.27 & \multirow{2}{*}{ a-b; a-c; b-c } \\
\cline { 2 - 5 } & Chicos y chicas & 169 & 789.88 & 223.78 & \\
\hline
\end{tabular}

$\mathrm{a}=$ Sólo chicos, $\mathrm{b}=$ Sólo chicas, $\mathrm{c}=$ Chicos $\mathrm{y}$ chicas 
Como puede verse, se encontraron diferencias estadísticamente significativos en el clima familiar deportivo $F_{(2,353)}=10.045, p=.000$, en función del sexo de los hijos de la unidad familiar. El análisis de comparaciones múltiples (post hoc) a través del test HSD de Tukey confirmó que estas diferencias se dieron entre los tres grupos analizados. Así la percepción del clima familiar deportivo fue más positiva $(\mathrm{p}<.05)$ en los adolescentes en cuyas familias sólo había chicos, frente a aquellas familias en las que había chicos y chicas; siendo la percepción más baja, la reportada por las adolescentes que eran hijas únicas o sólo tenían hermanas.

\section{DISCUSIÓN}

Los resultados obtenidos han confirmado lo aportado por la investigación previa tanto en cuanto al menor nivel de autoconcepto físico exhibido por las chicas (Esnaola, 2009a; Goñi et al., 2004; Goñi et al., 2006) como en cuanto a la relación directa y positiva entre el autoconcepto físico y sus subdominios específicos y la frecuencia de actividad físico-deportiva de los adolescentes (González y Alvariñas, 2004; Goñi et al., 2006; Moreno et al., 2007; Ruiz de Azúa, Rodríguez y Goñi, 2004). Si tomamos en cuenta además la menor frecuencia de actividad reportada por las chicas, puede apoyarse la consideración del autoconcepto físico y sus subdominios como variables determinantes de la elección de actividades físico-deportivas y/o de la motivación hacia las mismas; y, los efectos beneficiosos del ejercicio físico sobre el autoconcepto (Eccles y Harold, 1991; Esteve et al, 2005; Fox y Corbin, 1989; Moreno et al., 2007; Sonstroem, Speliotis y Fava, 1992).

Existe una excepción destacable a este respecto, en el caso de las chicas. La ausencia de relación significativa entre el atractivo físico y la frecuencia de participación en actividades físico-deportivas. Fox y Corbin (1989) ya afirmaron que existía una relación de menor intensidad entre el subdominio atractivo y los hábitos de ejercicio de la que se establecía con el resto de subdominios. Los trabajos realizados por los creadores del CAF por su parte, no registran esta diferencia (Goñi et al., 2006).

Parece por lo tanto que en el caso de las adolescentes, ni una mayor participación en actividades físico-deportivas tiene efectos positivos sobre la imagen física, ni en el caso de las chicas se ve el deporte como un medio para su mejora. El estereotipo actual de mujer atractiva no suele responder al estereotipo de mujer deportista (musculada, con ropa deportiva, etc.), aspecto que podría explicar esta circunstancia. En este sentido, Goñi et al. (2006) afirman que los mensajes verbales, los modelos y situaciones sociales, entre los que pueden encontrarse este tipo de imágenes estereotipadas sobre la mujer, presentan a menudo relaciones significativas con el subdominio atractivo del autoconcepto físico.

Por otra parte se ha podido observar que la influencia parental es fundamental para la adquisición del hábito deportivo. El interés por la actividad físico-deportiva varía de forma importante entre hombres y mujeres (Eccles et al., 1990; Fredricks y Eccles, 2004; Macías, 1999) y esta diferencia podría ser una consecuencia del proceso de socialización al que niños y niñas se ven sometidos desde la niñez temprana. No en 
vano, el valor otorgado a una actividad se encuentra fuertemente relacionado con la percepción de autocompetencia del sujeto en ese ámbito específico (Rodríguez, Wigfield y Eccles, 2003; Simpkins y Davis-Kean, 2005); y parece, a tenor de los resultados obtenidos, que en el desarrollo de estas autopercepciones podría tener una gran importancia la socialización familiar específica.

La adquisición de los estereotipos de género que podrían aparecer detrás de estas relaciones constituye una función de los procesos de socialización a través de los cuales determinadas instituciones sociales enseñan los valores propios de una sociedad dada (Macías, 1999) entre los que se encuentra el deporte. La familia, dada la extensa permanencia vital de los adolescentes en éste ámbito es una institución muy influyente en este tipo de procesos. Nuestros resultados indican, que chicos y chicas perciben de sus padres actitudes diferentes hacia la actividad físico-deportiva y más aún, que los adolescentes perciben un clima familiar deportivo más favorable, cuanto más chicos existen entre los hijos totales.

Pese a que no existen datos anteriores que relacionen la variable clima familiar deportivo con el sexo de los hijos e hijas, los resultados apoyan los presupuestos teóricos precedentes (Brustad, 1993; Eccles et al., 1990; Fredericks y Eccles, 2005; Horn y Horn, 2007; Jacobs y Eccles, 1992), en los que se asegura que la valoración de la actividad físico-deportiva y la proporción de actividades a los hijos por parte de los padres se encuentra afectada por el sexo de los hijos. Padres y madres se relacionan de forma diferente y proporcionan diferentes estímulos, materiales, juegos etc. a sus hijos e hijas (Roberts, 2001; Weiss, Smith y Theeboom, 1996) y, en definitiva, les exponen a actividades distintas muchas veces en concordancia con los roles de género, condicionando su bagaje de valores y su propia autopercepción.

Es destacable también que la percepción del clima familiar deportivo sólo se ha relacionado de forma significativa con la actividad físico-deportiva de las chicas. En este sentido, Rönkä y Poikkeus (2000) confirmaron que la influencia de los padres en los procesos de socialización deportiva era mayor en el caso de las chicas que en el de los chicos, resultados que confirman nuestros datos. Podría suceder que en el caso de los varones, la influencia del medio cultural, que habitualmente mantiene una imagen estereotípicamente masculina del deporte redujese el papel determinante de los padres y madres, cuyo apoyo sería fundamental entre las chicas.

La adolescencia es una etapa decisiva en lo que respecta a los hábitos físicodeportivos. Las tasas de sedentarismo se incrementan conforme avanza (Kemper, et al., 2001; Kimm et al., 2002; Crocker et al., 2006) y son especialmente preocupantes entre las chicas (Crocker et al. 2006; Mendoza, 2000). La participación deportiva se encuentra condicionada no sólo por las características personales del adolescente; los agentes de socialización y muy especialmente la familia tendrían un papel determinante (Bandura, 1984). Así, en el trabajo presentado, las actitudes de valoración y proporción de actividades físicas y deportes por parte de los padres, englobadas por el constructo clima familiar deportivo, se relacionaron con el autoconcepto físico de los chicos y chicas, variable central de la motivación deportiva y con su frecuencia de práctica. De igual modo, las percepciones paternas variaron en función del sexo de sus hijos e hijas. 
Estos resultados, pese a proceder del análisis de los datos de una muestra demasiado reducida como para poder considerarlos generalizables y confirmar las variaciones inter-grupos, se encuentran en sintonía con la investigación previa acerca de la importancia de la socialización deportiva familiar para la adquisición de un hábito saludable como es el deporte. La investigación previa y lo aquí concluido ponen de relieve la necesidad de analizar con un mayor detenimiento esta realidad y poner en marcha programas de formación específicos para padres y madres que permitan incrementar la experiencia físico-deportiva de los niños y adolescentes y hacer ésta más beneficiosa y satisfactoria.

\section{Agradecimientos}

Este artículo se ha realizado dentro del proyecto EDU2009-10102 (Subprograma EDUC) subvencionado por el MICINN y con la colaboración del Programa de Formación Personal Investigador PIFA01/2007/024 (UPV/EHU).

\section{REFERENCIAS}

Amezcua, J.A. y Pichardo, M.C. (2001). Diferencias de género en autoconcepto en sujetos adolescentes. Anales de Psicología, 16(2), 207-214.

Bandura, A. (1984). Teoría del aprendizaje social. En J. Torregrosa y E. Crespo (Eds.), Estudios básicos de Psicología Social (pp. 213-224). Barcelona: Biblioteca Hora. Centro de investigaciones sociológicas.

Boyd, K.R. y Hrycaiko, D.W. (1997). The effect of a physical activity intervention package on the self-esteem of preadolescent and adolescent females. Adolescence, 32(127), 693-709.

Brettshneider, W.D. y Heim, M. (1997). Identity, Sport and youth development. En K.R. Fox (Ed.), The physical self: from motivation to well being (pp. 205-227). Champaign, Illinois: Human Kinetics.

Brustad, R.J. (1992). Integrating socialization influences into the study of children's motivation in sport. Journal of Sport and Exercise Psychology, 14, 59-77.

Brustad, R.J. (1993). Who will go out and play? Parental and psychological influences on children's attraction to physical activity. Pediatric Exercise Science, 5, 210-223.

Brustad, R.J. (1996). Attraction to physical activity in urban schoolchildren. Parental socialization and gender influences. Research Quarterly for Exercise and Sport, 67(3), 316-323.

Crocker, P.R.E., Sabinston, C.M., Kowalsky, K.C., McDonough, M.H. y Kowalsky, N. (2006). Longitudinal Assessment of the relationship between Physical Self-concept and health related behaviour and emotion in adolescent girls. Journal of Applied Sport Psychology, $18,185-200$.

Eccles, J.S. y Harold, R.D. (1991). Gender differences in sport involvement: Applying the Eccles' Expectancy-Value Model. Journal of Applied Sport Psychology, 3, 7-35.

Eccles, J.S., Jacobs, J.E. y Harold, R.D. (1990). Gender role stereotypes, expectancy effects and parents's socialization of gender differences. Journal of Social Issues, 46(2), 183-201.

Escartí, A., García Ferriol, A. y Musitu, G. (1993). Desarrollo y validación estadística de una escala de medida del clima familiar deportivo. IV Congreso Nacional de Psicología de la Actividad Física y el Deporte. Sevilla.

Esnaola, I. (2009). Diferencias de sexo en el autoconcepto físico durante el ciclo vital. Psicología Conductual, 2, 365-380. 
Esnaola, I. y Zulaika, L.M. (2009). Physical activity and physical self-concept in a sample of middle-aged Basque adults. Perceptual and Motor Skills, 108, 479-490.

Esteve, J.V. (2005). Estilos parentales, clima familiar y autoestima física en adolescentes. (Tesis Doctoral). Valencia: Universidad de Valencia. Departamento de Psicología Social.

Esteve, J.V., Musitu, G. y Lila, M. (2005). Autoconcepto físico y motivación deportiva en chicos y chicas adolescentes. La influencia de la familia y de los iguales. Escritos de Psicología, 7, 82-90.

Forehan, R. y Nousiainen, S. (1993). Maternal and paternal parenting: critical dimensiones in adolescent functioning. Journal of Family Psychology, 7, 213-221.

Fredricks, J.A. y Eccles, J.S. (2004). Parental influences on youth involvement in sports. En M. Weiss (Ed.), Developmental sport and exercise psychology: a lifespan perspectiva (pp. 145-164). Morgantown, WV: Fitness Information Technology.

Fredricks, J.A. y Eccles, J.S. (2005). Family socialization, gender, and sport motivation and involvement. Journal of Sport and Exercise Psychology, 27, 3-31.

Fox, K.R. (2000). Self-esteem, self perceptions and exercise. Internacional Journal of Sport Psychology, 31, 228-240.

Fox, K.R. y Corbin, C.B. (1989). The physical self-perception profile: development and preliminary validation. Journal of Sport and Exercise Psychology, 11(4), 408-430.

González, M.A. y Alvariñas, M. (2004). Relación entre la práctica físico-deportiva extraescolar y el autoconcepto físico en la adolescencia. Revista de Educación Física: Renovar la Teoría y la Práctica, 94, 5-8.

Goñi A., Ruiz de Azúa, S. y Rodríguez, A. (2004). Deporte y autoconcepto físico en la preadolescencia. Apunts: Educación Física y Deportes, 77, 18-24.

Goñi A., Ruiz de Azúa, S. y Rodríguez, A. (2006). Cuestionario de autoconcepto físico (CAF). Manual. Madrid: EOS.

Goñi, A. y Zulaika, L.M. (2000). La participación en el deporte escolar y el autoconcepto en escolares de 10-11 años de la provincia de Guipúzcoa. Apunts: Educación Física y Deportes, 59, 6-10.

Harter, S. (1986). Processes underlying the construction, maitenance and enhancement of the selfconcept in children. En J. Suls y A. G. Greenwald (Eds.), Psychological perspectives of the self (Vol 3, pp. 137-181). Hilldsdale, N.J: Lawrence Erlbaum.

Horn, T.S. y Harris, A. (1996). Perceived competence in young athletes: Research findings and recommendations for coaches and parents. En F.L. Smoll y R.E. Smith (Eds.), Children and youth in sport: a biopsychosocial perspectiva (pp. 309-329). Madison, WI: Brown and Benchmark.

Horn, T.S. y Horn, J. (2007). Family influences on children's sport and physical activity participation, behaviour, and physical responses. En G. Tenenbaum y R. Eklund (Eds.), Handbook of Sport Psychology (pp. 685-711). Hoboken: Wiley and Sons.

Infante, G. y Goñi, E. (2009). Actividad físico-deportiva y autoconcepto físico en la edad adulta. Revista de Psicodidáctica, 14(1), 49-61.

Jacobs, J.E. y Eccles, J.S. (1992). The impact of mothers' gender-role stereotypic beliefs on mothers' and children's ability perceptions. Journal of Personality and Social Psychology, 63, 932-944.

Kemper, H., Twisk, J., Koppes, L., Van Mechelen, W. y Bertheke Post, G. (2001). A 15-year physical activity pattern is positively related to aerobic fitness in young males and females (13-27 years). European Journal of Applied Physiology, 84, 395-402.

Kimm, S., Glynn, N., Kriska, A., Barton, B., Kronsberg, S., Daniels, S., Crawford, P., Sabry, Z. y Liu, K. (2002). Decline in physical activity in black girls and white girls during adolescence. New England Journal of Medicine, 347, 709-715. 
Macías, M.V. (1999). Estereotipos y deporte femenino. La influencia del estereotipo en la práctica deportiva de niñas y adolescentes (Tesis Doctoral). Granada: Universidad de Granada.

Mendoza, R. (2000). Diferencias de género en los estilos de vida de los adolescentes españoles: Implicaciones para la promoción de la salud y para el fomento de la actividad físicodeportiva. En Fete-UGT (Ed.), Educación Física y Salud, Actas del Segundo Congreso Internacional de Educación Física (pp. 765-790). Cádiz: Publicaciones del Sur.

Moreno, J.A., Moreno, R. y Cervelló, E. (2007). El autoconcepto físico como predictor de la intención de ser físicamente activo. Psicología y Salud, 17(2), 261-267.

Pérez, V. y Devis, J. (2003). La promoción de la actividad física relacionada con la salud. La perspectiva de proceso y de resultado. Revista Internacional de Medicina y Ciencias de la Actividad Física y el Deporte, 3(10), 69-74.

Roberts, G.C. (1995). Motivación en el deporte y el ejercicio físico. Bilbao: Descleé de Bouwer.

Roberts, G.C. (2001). Advances in motivation in sport and exercise. Champaign ILL: Human Kinetics.

Rodríguez, T. y Beato, L. (2002). Factores relacionados con la imagen corporal. Un estudio longitudinal. Psiquiatría.com/Interpsiquis.2002. Disponible online en http://www.psiquiatria.com.

Rodríguez, D., Wigfield, A. y Eccles, J.S. (2003). Changing competence perceptions, changing values: Implications for youth sport. Journal of Applied Sport Psychology, 15, 67-81

Román, B., Serra, L., Ribas, L., Pérez-Rodrigo, C. y Aranceta, J. (2006). Actividad física en la población infantil y juvenil española en el tiempo libre. Estudio enKid (1998-2000). Apunts: Medicina del Deporte, 151, 86-94.

Rönkä, A. y Poikkeus, A.M. (2000). The effect of parenting and the quality of the parent-child relation on the well-being of boys and girls in early adolescence. The Finnish Journal of Youth Research, 18, 3-18.

Ruiz de Azúa, S., Rodríguez, A. y Goñi, A. (2004). Deporte y autoconcepto físico en la preadolescencia. Apunts: Educación Física y Deportes, 77, 18-24.

Sallay, H. (2000). The role of the family in shaping self-concept and cognitive styles in Hungary. Research Support Scheme Electronic Library. Disponible online en http://elib.rss.cz/diglib/pdf/94.pdf

Simpkins, S.D. y Davis-Kean, P.E. (2005). The intersection between self-concepts and values: links between beliefs and choices in high school. New Directions for Child and Adolescent Development, 110, 31-47.

Sonstroem, R.J., Speliotis, E.D. y Fava, J.L. (1992). Perceived physical competence in adults: an examination of the physical self-perception profile. Journal of Sports and Exercise Psychology, 14, 207-221.

Weiss, M.R. (2000). Motivating kids in physical activity. President's Council on Physical Fitness and Sports Research Digest, 3(11), 1-8.

Weiss, M.R., Smith, A.L. y Theeboom, M. (1996). "That's what friends are for": Children's and teenagers' perceptions of peer relationships in the sport domain. Journal of Sport and Exercise Psychology, 18, 347-379.

Recibido: 1 de marzo de 2010

Recepción Modificaciones: 19 de agosto de 2010

Aceptado: 29 de agosto de 2010 